\title{
CEBAF UPGRADE CRYOMODULE COMPONENT TESTING IN THE HORIZONTAL TEST BED (HTB)*
}

\author{
I. E. Campisi ${ }^{\dagger}$, B. Carpenter, G. K. Davis, J. Delayen, M. A. Drury, E. Feldl, J. Fischer, A. Guerra, \\ T. Hiatt, C. Hovater, K. Macha, J. Mammosser, V. Nguyen, H. L. Phillips, J. Preble, \\ TJNAF, Newport News, VA 23606, USA
}

\begin{abstract}
The planned upgrade of the CEBAF electron accelerator includes the development of an improved cryomodule. Several components differ substantially from the original CEBAF cryomodule; these include: the new 7-cell, $1.5 \mathrm{GHz}$ cavities with integrated helium vessel, a new, backlash-free cavity tuner, the waveguide coupler with its room-temperature ceramic window. In order to test the design features and performance of the new components, a horizontal cryostat (Horizontal Test Bed) has been constructed which allows testing with a turn around time of less than three weeks. This cryostat provides the environment for testing one or two cavities, with associated auxiliary components, in a condition similar to that of a real cryomodule. A series of tests has been performed on a prototype 7-cell cavity and the above-mentioned systems. In this paper the results of the tests on the cryostat, on the cavity performance, on its coupler, on the tuner characteristics, and on the microphonics behavior are presented.
\end{abstract}

\section{INTRODUCTION}

As a part of the plans for the Energy Upgrade of the CEBAF accelerator [1], several components of the new Cryomodule have been redesigned and some new solutions adopted for the first time. Before the process of construction of the cryomodules begins, the hardware had to be tested to determine their validity as an integrated new design. A small horizontal cryostat was designed and assembled which allowed testing of a cavity (Figure 1) with all the auxiliary components with turn around times much faster than for a full cryomodule.

With this cryostat a series of tests was performed and the critical components characterized; below, the main results are reported.

\section{MECHANICAL AND VACUUM DESIGN}

The HTB cryostat is made from a modified CEBAF $1 / 4$ cryomodule (Figure 2). In order to accommodate the two longer 7 cell cavities, the cryostat was extended, as were the vacuum vessel and thermal and magnetic shieldings. The cavities are supported on an aluminum strong back mounted in the cryostat using rod supports similar to the CEBAF cryostat. The cavities are located inside the cryostat so that the existing top hat penetration in the vacuum vessel can be used. This minimizes the cryostat rework required and preserves the warm window RF

\footnotetext{
* Supported by US DOE Contract No. DE-AC05-84-ER40150

†campisi@jlab.org
}

interface position. Initially the Horizontal Test Bed was configured with two dummy cavities consisting of a pipe within a pipe. These were design to approximate the cavity assemblies in size, volume, and flow pattern. Subsequent cooldowns included a single cavity assembly for testing and a helium pot to maintain similar inventory levels through all tests. Cavity vacuum is extended through the end can to room temperature on one end where an ion pump is mounted.

\section{CRYOGENICS}

The HTB has a 2 Kelvin primary and 50 Kelvin shield helium circuit. The primary circuit uses a $3 / 4$ in. supply header which feeds the 2 helium vessels and 2 redundant liquid level sensor stand pipes in parallel. The 4 in. return header is similarly configured. Cryogens enter and exit the cryostat through existing prototype CEBAF end cans. Several cooldowns of the HTB were performed. Helium process instrumentation confirmed anticipated cooldown profiles that include the simultaneous cooling of both the cavity and dummy helium vessel. Refrigerator stability and transfer line pressure fluctuations hampered the accuracy of some of the measurements described below.

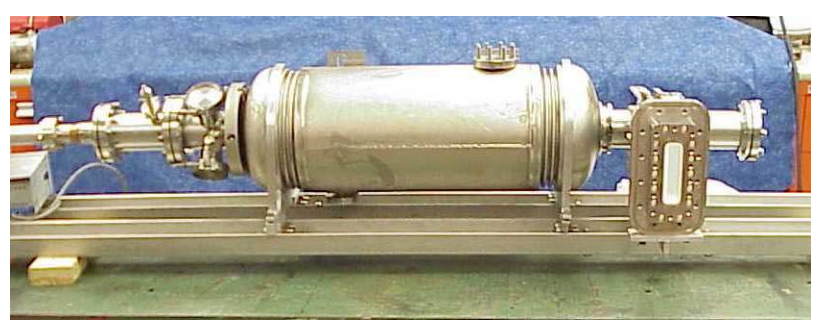

Figure 1: The 7-cell cavity with the integrated helium vessel and waveguide coupler and alumina window.

\section{CAVITY TESTING}

The new design 7-cell cavity with integrated Helium vessel was tested in the horizontal configuration. The $\mathrm{Q}_{\mathrm{ext}}$ of the waveguide fundamental power coupler was measured to be $3.8 \times 10^{7}$ about a factor of two larger that the design value. Testing with a $2 \mathrm{~kW}$ klystron allowed us to reach a maximum gradient of $11 \mathrm{MV} / \mathrm{m}$, consistent with the measurements performed in the vertical cavity configuration.

With the integrated helium vessel the amount of stored helium is considerably smaller than in the old CEBAF cryomodule and the ability to measure the unloaded $\mathrm{Q}$ calorimetrically via helium mass flow was impeded by the rapid decrease of the liquid level, so no reliable value for the unloaded $Q$ was estimated. 


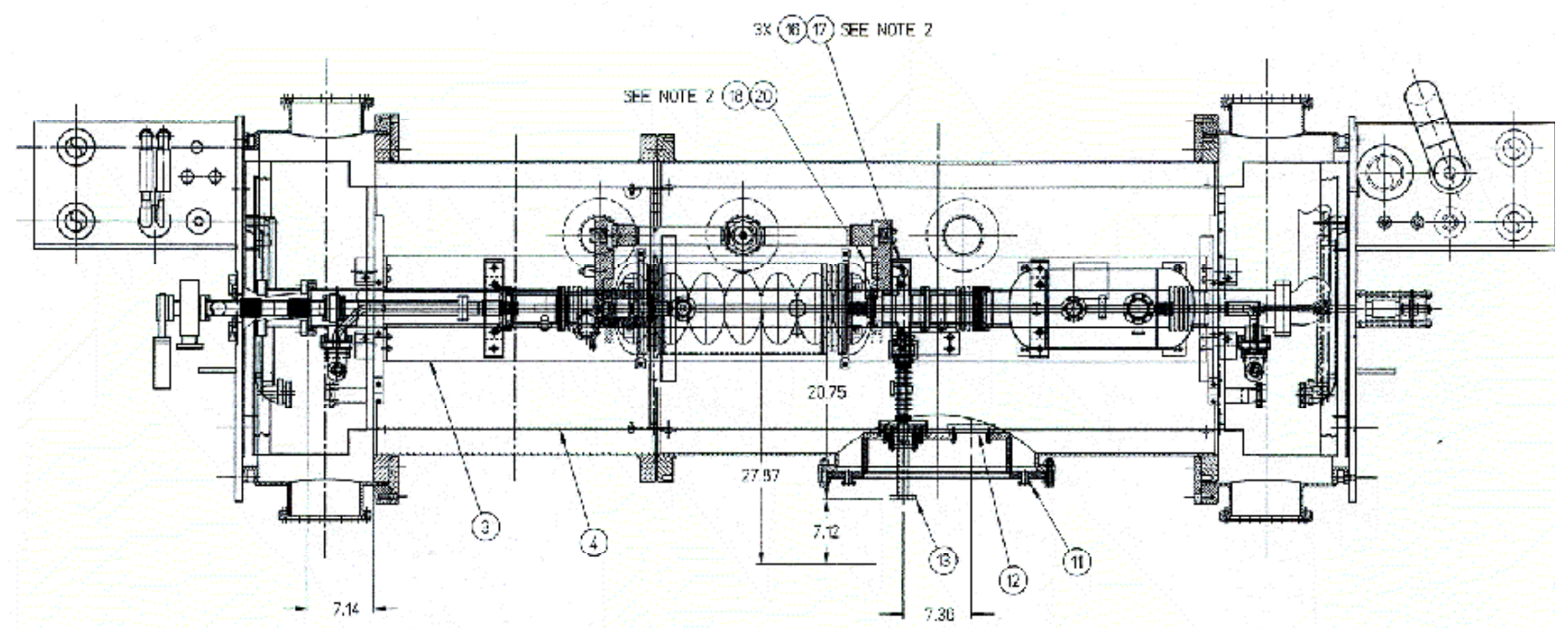

Figure 2: Layout of the HTB cryostat (top View) with a 7-cell cavity and waveguide fundamental power coupler.

\section{TUNER}

The design of the CEBAF Upgrade tuner has been described elsewhere [2]. The novel design includes a scissors-jack construction, which is operated under tension, thus eliminating all possible backlash and hysteresis (Figure 3).

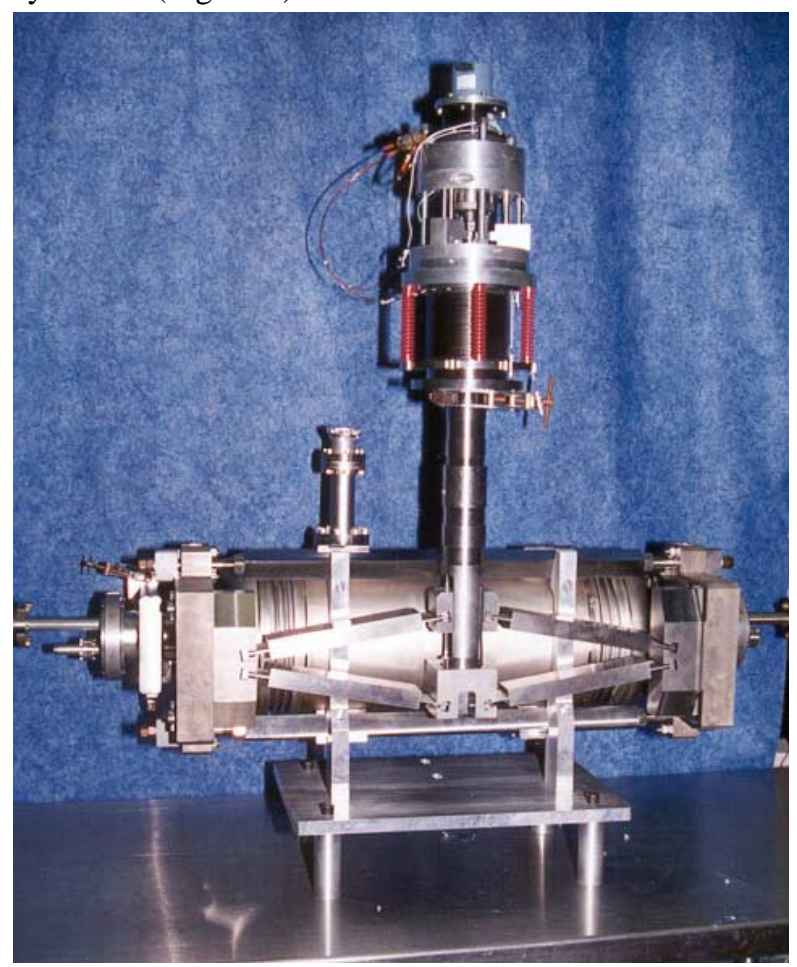

Figure 3: The tuner and cavity assembly.

Tuner testing was performed with the cavity at $2 \mathrm{~K}$ in the Horizontal Test Bed [3]. The cavity was locked to its resonant frequency with a phase locked loop (PLL).
Tuner range and hysteresis were measured with a frequency counter. Tuner resolution was measured using a Cavity Resonance Monitor instrument, a custom-built IQ demodulator, which provides a voltage output proportional to the difference in frequency between the PLL output and a reference local oscillator. In addition to testing these requirements, tests were performed on both tuners to analyze possible tuner-induced microphonics. Microphonics-induced frequency shifts measured were less than approximately $0.3 \mathrm{~Hz}$ peak above background.

Finally the frequency response of the fine tuner was measured. Although the fine tuner was not designed for the purpose, there is interest in the possibility of using the tuner to compensate for Lorentz detuning during pulsed operation, or to actively cancel cavity frequency shifts caused by structure-borne vibrations (microphonics). The amplifier used to drive the piezo actuators limited the system response, which was nonetheless judged to be usable (magnitude $>17.4 \mathrm{~Hz}$ peak-to-peak) for the input frequency range from $\mathrm{DC}$ to $100 \mathrm{~Hz}$.

\section{MICROPHONICS}

Upgrade cryomodule microphonics testing was performed in the HTB with the cavity at $2 \mathrm{~K}$, and RFlocked to a PLL [4]. The tests accomplished included response to background vibration, swept sinusoidal excitation, high-power RF pulse excitation, mechanical impulse excitation. The resulting cavity frequency shifts were measured in two ways. One method was via the PLL error signal. The PLL error signal is a voltage that is proportional to the difference in frequency between the cavity transmitted power feedback signal and the PLL error loop setpoint. The second method used was the CRM instrument (see Tuner Testing 
above). The system specification requires less than 3.5 $\mathrm{Hz}$ rms detuning due to microphonics, actual measurements showed $2.5 \mathrm{~Hz}$ rms. A typical background excitation measurement, made with the cavity operating at high power, is shown in Figure 4. The peak at $33.7 \mathrm{~Hz}$ was identified as a prominent system resonance, excited by broadband noise and showing up in both the mechanical impulse and swept sine responses also. The peak at $54.7 \mathrm{~Hz}$ corresponds to a peak in the ambient ground spectrum, but no system resonance at this frequency was measured.

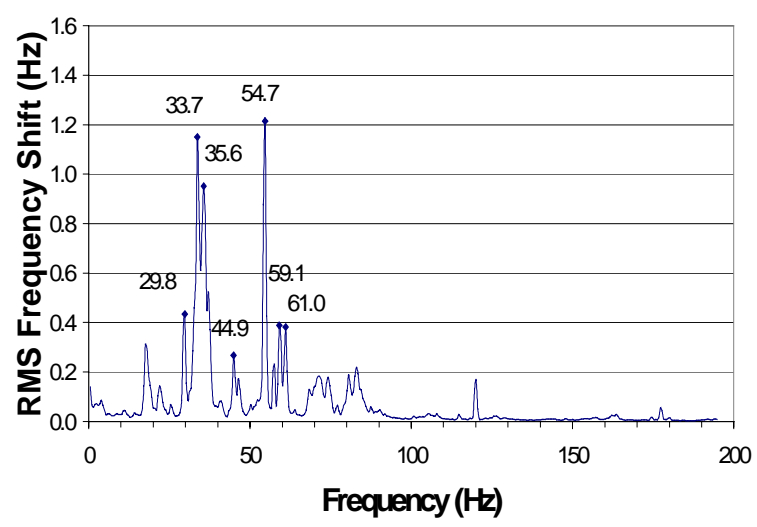

Figure 4: Results of the microphonics measurements performed on the upgrade 7-cell cavity in the HTB.

\section{FUNDAMENTAL POWER COUPLER}

\subsection{RF properties}

Although similar in concept to the original CEBAF cavity waveguide coupler, the Upgrade coupler waveguide is less sensitive to tolerances and does not impart a transverse kick, as the original one did [5].

It incorporates a 1/4 stub with symmetrical fields and the coupling strength is solely determined by the distance of the waveguide from the cavity's end cell's evanescent fields.

The coupler's assembly's external Q was measured in different waveguide conditions and a value of 3.8 was obtained.

\subsection{Thermal properties}

The thermal properties of the coupler's design are also discussed in [5]. The stainless steel waveguide is copper electroplated (in the prototype system with a 3 $\mu \mathrm{m}$ layer) was optimized both for static and dynamic heat load. The measurements performed on the waveguide temperature profile with and without about $1.7 \mathrm{~kW}$ input power closely follow the calculated values [6].

\section{HOM}

The new 7-cell cavity for the CEBAF Upgrade does not include Higher-Order-Mode extraction waveguides as in the original 5-cell design. More compact HOM filters are being considered, following the DESY TESLA developments [7].

The first test performed on the prototype cavity was done without the filters, in order to determine the natural damping of the modes through fundamental power waveguide and beam pipe. Generally, Q's of the order of $10^{5}-10^{6}$ have been observed in the $\mathrm{TE}_{111}$ and $\mathrm{TM}_{110}$ dipole passbands. Self-polarization of some modes due to the perturbation of the fundamental power coupler shows occasional increase of one of the dipole modes to the $10^{7}$ range. These values are generally considered adequate for the operation of the CEBAF Upgrade.

\section{CONCLUSIONS}

The Horizontal Test Bed cryostat, build to accommodate the testing of critical components of the CEBAF Upgrade cryomodule, has been successfully employed to test upgrade tuner, fundamental power coupler, 7-cell cavities and their behavior under realistic conditions.

\section{ACKNOWLEDGEMENTS}

The authors would like to thank $\mathrm{H}$. Whitehead, K. Worland, F. Humphry, J. Brock, J. Takacs, T. Elliott, C. Hutton and the cryogenics Group for support in the design, assembly and testing of the HTB.

\section{REFERENCES}

[1] J. R. Delayen et al., "Cryomodule Development for the CEBAF Upgrade," PAC99, New York, NY, April 1999.

[2] J. R. Delayen et al., "Frequency Tuning of the CEBAF Upgrade Cavities," PAC99, New York, NY, April 1999.

[3] G. K. Davis et al., "Development and Testing of a Prototype Tuner for the CEBAF Upgrade Cryomodule," PAC2001, Chicago, IL, June 2001.

[4] G. K. Davis et al., "Microphonics Testing of the CEBAF Upgrade 7-Cell Cavity," PAC2001, Chicago, IL, June 2001.

[5] J. R. Delayen et al., "An RF Input Coupler System for the CEBAF Energy Upgrade Cryomodule," PAC99, New York, NY, April 1999.

[6] T. Hiatt et al., "Cryogenic testing of the RF input waveguide for the CEBAF Upgrade Cryomodule," PAC2001, Chicago, IL, June 2001.

[7] J. Sekutowicz, TTF Design Report, p.120, Hamburg, Germany, March 1995. 